\title{
TOXOCARIASIS: VISCERAL AND OCULAR LARVA MIGRANS
}

\section{By}

\section{TOSSON A. MORSY}

Department of Parasitology, Faculty of Medicine, Ain Shams University, Cairo 11566, Egypt (tossonmorsy@med.asu.edu.eg.or morsyegypt2014@gmail.com) Abstract

Toxocariasis is a neglected socioeconomically important zoonotic nematode parasite that afflicts millions of the pediatric and adolescent populations worldwide, especially in impoverished communities. This disease is caused by infection with the larvae of Toxocara canis and $T$. cati, the most ubiquitous intestinal nematode parasite in dogs and cats, respectively. Human infections can lead to syndromes called visceral larva migrans (VLM), ocular larva migrans, neurotoxocariasis, and covert toxocariasis. Infection is not often fatal, but the inflammatory response to migrating larvae is associated with increased leukocytosis, including generalized lymphadenopathy, endophthalmitis, granulomatous hepatitis, asthma, endomyocarditis, and high eosinophilia ( $>30 \%)$ as well as malignancy

\section{Introduction}

Toxocariasis (also called visceral larva migrans or VLM) refers to human infection caused by roundworms that are not natural human parasites. Toxocariasis occurs as a result of human infection with the larvae of the dog ascarid, Toxocara canis, and/or, less commonly, the cat ascarid, Toxocara cati. Another form of VLM is caused by human ingestion of eggs of the pig ascarid, Ascaris suum. Clinical presentations consist of VLM and ocular larva migrans (OLM); infection may also be subclinical (Won et al, 2008). Toxocariasis occurs worldwide. Infection tends to occur more frequently in tropical regions than in temperate regions, and more frequently among rural populations than urban populations. Toxocara larvae can develop at temperatures $<50$ degrees Fahrenheit although efficiency decreases as the temperature increases (Azam et al, 2012). In the United States, the seroprevalence of Toxocara has been estimated at $13.9 \%$; rates are increased among individuals living in poverty and among certain minority groups (especially African Americans). Prevalence rates of $40 \%$ or more have been reported in Indonesia and Brazil (CDC, 2011). In North America, it is estimated that about $5 \%$ of dogs and puppies are infected (Mohamed et al, 2009). In Egypt, like other developing countries, the risk of zoonotic infection related to domiciled, as well as stray dogs is high due to keeping of livestock and pets inside houses in most rural areas (Youssef and Uga, 2014).
Dogs and foxes are the reservoir for $T$. $c a-$ nis, but puppies and cubs pose the greatest risk of spreading the infection to humans (CDC, 2009). Infection is characterized by encysted second stage larvae. Larvae can become reactivated in pregnant females and cross the placental barrier to infect pups. Vertical transmission can also occur by breastmilk (CDC, 2006). In the environment, shed eggs embryonate and become infective after about three weeks and humans acquire infection as accidental hosts. Following ingestion of infective eggs by dogs or cats, they hatch to larvae $(0.5 \mathrm{~mm})$ penetrate the gut wall and migrate through the lungs, bronchial tree, and enter the esophagus; adult worms develop in the small intestine, where they lay eggs that are shed in the stool. In the older animals, larvae penetrate the gut wall and subsequently larvae encyst in tissues. Encysted larvae can reactivate in female dogs during late pregnancy and infect puppies by transplacental and trans-mammary routes; adult worms can subsequently become established in the small intestines of puppies. Eggs ingested by small non-canine mammals can hatch and larvae can penetrate gut wall with subsequent migration into various tissues where they encyst (Despommier, 2003). Humans acquire infection via ingestion of embryonated/ larvated eggs in soil or contaminated food, or by ingestion of encapsulated Toxocara larvae in improperly cooked tissues of infected paratenic hosts, such as the rabbits, cattle, sheep, goats and 
chickens (Macpherson, 2013). Humans are considered as an accidental or aberrant host, therefore, Toxocara larvae cannot develop into adult worms inside the human body (Zibaei, 2017). Direct contact with infected puppies and kittens is not classically considered to be risky for human infection since the eggs must embryonate before becoming infective, although, sometimes pets carry embryonated eggs in their fur (El-Tras et al, 2011). After the eggs pass into the environment, it takes about 2 to 4 weeks for infective larvae to develop in the eggs. If a person ingests one of these infective eggs, then they can become infected with toxocariasis. Following ingestion, the eggs hatch and larvae penetrate the intestinal wall and are carried by circulation to various tissues as liver, heart, lungs, brain, muscle, eyes (Lim, 2008). The larvae do not undergo any more development in these sites, but the host inflammatory response against the migrating larvae can cause both mechanical and immunopathological damage to tissues, which leads to severe local reactions that are the basis of toxocariasis. However, toxocariasis is not spread by person-to-person contact like a cold or the flu (CDC, 2019).

Clinically Toxocara infections are often associated with considerable variability in presentation. Because T. canis larvae migrate to various body organs, such as the liver, heart, lungs, kidneys, brain, muscle and eyes, a broad range of clinical symptoms can be developed (Fan et al, 2015). There are four types of disease: ocular larva migrans (OLM), visceral larva migrans (VLM), neurotoxocariasis, and covert toxocariasis. The covert disease most often presents with simple, persistent eosinophilia and may be attributed to continuation of migratory phase (Nicoletti, 2013). Interestingly, this migratory phase may last for years (Woodhall and Fiore, 2014). The disease severity depends on parasite burden, duration of larval migration, and age- and immune-mediated responses of the affected individuals (Moreira et al, 2014). Visceral larva migrans (VLM) is principally a disease of young children, especially those with exposure to playgrounds and sandboxes contaminated by dog or cat feces (Carvalho and Rocha, 2011). VLM are a consequence of both the damage caused by migrating larvae and the host eosinophilic granulomatous response. Migration of larvae can cause eosinophilic infiltration, granuloma formation, or eosinophilic abscesses. In young children VLM resulted in hepatitis \& pneumonitis as the larvae migrate through the liver and lungs, respectively. Heavy infection may result in fever, anorexia, malaise, irritability, hepatomegaly, respiratory symptoms, pruritic urticaria-like cutaneous lesions and eosinophilia (Beshear and Hendley, 1973).

Larvae frequently localize in the liver; hepatic manifestations may include hepatomegaly or nodular lesions. Pulmonary involvement may cause dyspnea, wheezing, and a chronic nonproductive cough in 20 to $80 \%$ of patients (Snyder, 1961). Roles are common on physical examination. The chest radiograph demonstrates abnormalities in $\geq$ $40 \%$ of patients with symptomatic illness (Walsh, 2011). Bilateral peribronchial infiltration is most common; parenchymal infiltrates can also occur (Sakai et al, 2006). Computed tomography (CT) may demonstrate multifocal subpleural nodules with halo or ground-glass opacities and ill-defined margins, but severe respiratory tract involvement was an uncommon complication in heavy infection (Ota et al, 2009).

Toxocariasis can be mistaken for metastatic diseases; the presence of eosinophilia and radiologic findings may be helpful differentiating features (Marx et al, 2007). Larvae can also travel via the systemic circulation to the muscles, heart, eye, and/or central nervous system (Jabbour et al, 2011). Manifestations of CNS include eosinophilic meningoencephalitis, space occupying lesions, myelitis, and cerebral vasculitis causing seizures. Manifestations of the peripheral nervous system include radiculitis, affection of the cranial nerves, or musculoskeletal involvement. 
Sander (2003) in United Kingdom reported that malaria, toxoplasmosis and toxocariasis can cause not only acute symptomatic seizure but also remote symptomatic epilepsy. Myocardial \&/or CNS involvement was rarely fatal (Good et al, 2004).

Ocular larva migrans: OLM involvement may occur as the sole VLM manifestation; it often presents in individuals without antecedent history of symptomatic VLM. OLM occurs most commonly among older child ren and adults (Stewart et al, 2005). Berrett et al. (2017) found an overall Toxocara seroprevalence of $5.1 \%$. Increasing age, male sex, low educational attainment, low income, and immigration status each was associated with Toxocara seropositivity. Mexican Americans had reduced odds of exposure. They concluded that exposure to Toxocara continues in the United States and that several demographic factors influence the risk of exposure. The ocular lesion is due to larval localization in the eye and the granulomatous response around the larva. Common symptoms are unilateral visual impairment, causing failing vision and subsequent strabismus. The typical lesion is a whitish elevated granuloma. OLM may present as uveitis, papillitis, or endo-phthalmitis (Chuah et $a l, 2006)$. Ocular lesions may resemble retinoblastoma. The most serious infection is retina invasion with granuloma formation in periphery or posterior pole, leading to retina dragging and eventual retinal detachment that can lead to blindness (Suh et al, 2006).

Jones et al. (2007) in USA reported that multivariate analysis controlling demographic and risk factors showed that persons infected with Toxocara spp. were more likely to be infected with Toxoplasma gondii (odds ratio $[\mathrm{OR}]=1.93,95 \% \mathrm{CL}=1.61-2.31$ ), and $T$. gondii infected persons were more likely to be infected with Toxocara spp. (OR = $1.91,95 \% \mathrm{CL}=1.59-2.28$ ). They added that infection with $T$. gondii and Toxocara spp. were common and could be prevented by many similar interventions. Guneratne et al. (2011) in Sri Lanka reported the co-infection of T. gondii, Toxocara spp. and tuberculosis in a child with chronic lymphadenopathy and eosinophilia.

Other presentations: Toxocara can also present with other manifestations. Mild infection may present with eosinophilia only. Other symptoms may include fever, headache, behavioral disturbances, anorexia, abdominal pain, rash, hepatomegaly, vomiting, nausea, as well as wheezing and pulmonry infiltrates (Cooper, 2009).

Wheezing and pulmonary infiltrates, together with eosinophilia, are also the hall-mark features of childhood asthma. It has been postulated that the presence of

Toxocara larvae in lungs may be an underlying factor in onset of allergic pulmonary disease, perhaps because of the host response to the parasite; a causal association remains uncertain. Chan et al. (2001) reported relationship between exposure to Toxocara infection and bronchial asthma in Malaysian children warrants further evaluation. A study done in the United States found a significant association between prior toxocaraisis and diminished pulmonary function as measured by forced expiratory volume in one second FEV (Muñoz-Guzmán et al, 2010). However, other studies failed to demonstrate an association between Toxocara seropositivity and asthma; one study suggested that this association may be attributed to cross-reactivity. There was also some evidence that treatment of chronic cough with both asthma therapy and anthelminthic may be beneficial (Bede et al, 2008).

Cancer: Parasites infect about 2 billion people worldwide with parasitic nematodes, causing more than one billion people to suffer tremendous pain and massive economic loss. Anderson et al. (2006) in USA reported a 22-month-old girl with a renal mass had multiple small pulmonary nodules on $\mathrm{CT}$ at her initial presentation. After biopsy and neo-adjuvant chemotherapy, a Wilms tumor was resected and pulmonary nodules were regressed on the CT. They concluded that toxocariasis must be considered as a 
differential diagnostic possibility for multiple liver lesions and multifocal peripheral pulmonary opacities in young children with Wilms tumor. Hotez and Wilkins (2009) in USA reported that $T$. canis as an important roundworm of canids and a fearsome animal parasite of humans. Kang et al. (2014) in Korea reported a 62-year-old woman presented with diffuse bladder wall thickening and multiple liver masses with peripheral eosinophilia and urinary symptoms. They considered malignancy or eosinophilic cystitis via clinical manifestations and imaging findings. However, no suspicious malignant lesions were observed on cystoscopy and liver mass biopsy revealed the presence of eosinophilic necrotizing granuloma without malignant cells. Kim et al. (2018) in Korea reported that the endoscopic biopsy from a patient colon revealed a tubulovillous adenoma. Malignancy was not ruled out, so the researchers decided to perform a radical colectomy, but VLM must be included in the differential diagnosis as a cause of intraperitoneal tumors.

Diagnosis: In general, visceral larva migrans (VLM) should be suspected in setting of compatible clinical manifestations, together with leukocytosis, eosinophilia, and hypergammaglobulinemia (elevated serum levels of $\operatorname{IgE}, \operatorname{IgG}, \& \operatorname{IgE})$. Marked leukocytosis with eosinophilia occurs in more than $30 \%$ of cases, and elevated titers of anti-A or antiB-isohemagglutinins were commonly observed in $50 \%$ of patients (Cypess et al, 1977). The eosinophilic granulomatous hepatitis may develop leading to abnormalities in liver function tests, including elevated transaminases and/or alkaline phosphatase.

ELISA antibody assay detects human IgG antibodies to Toxocara excretory/secretory antigens (Jones et al, 2008). The test can detect subclinical or mild infection, though it cannot differentiate between $T$. canis and $T$. cati infections. For VLM and some forms of covert toxocariasis, the sensitivity and specificity of the Toxocara EIA (titer 1:32) were estimated at $78 \%$ \& $92 \%$, respectively (Ma- gnaval et al, 1991). A positive ELISA result does not necessarily indicate the presence of active Toxocara infection or prove that clinical symptoms are attributable to that toxocariasis; the result must be interpreted in the setting of compatible clinical symptoms and epidemiologic exposure. Cross-reactivity of ELISA assay with other parasite antigens is common, and the test may remain positive for several years even following treatment. In some settings, positive ELISA results can be confirmed by western blotting. Definitive diagnosis of VLM may also be established by detection of larvae in biopsy tissue which showed Toxocara larvae within eosinophilic granulomatous lesions. But, biopsy was rarely indicated (Despommier, 2003). The sensitivity of ELISA for ocular larva migrans (OLM) is considerably lower than for VLM; the diagnosis of OLM generally relies on the findings on ophthalmologic examination (de Visser et al, 2008). It was possible to compare antibody levels between serum and aqueous humor; if the (level of specific IgG in aqueous humor/level of specific IgG in serum/total IgG in aqueous humor/ total IgG in serum) was greater than 3.0, this can be considered diagnostic (Fogt-Wyrwas et al, 2007).

PCR-based methods for detecting Toxocara in clinical samples have been described, but are not commercially available (Roig et al, 1992).

Stool examinations are not helpful since the parasite does not complete a full life cycle involving the human gastrointestinal tract. Pulmonary involvement may result in eosinophilia that is detectable in bronchoalveolar lavage (BAL) fluid. One marked pulmonary infiltration case showed $64 \%$ eosinophils in the BAL analysis (Eberhardt et al, 2005).

In the setting of CNS involvement, the cerebrospinal fluid showed eosinophils (Ishibashi et al, 1992). Eosinophilic meningoencephalitis is based on clinical manifestations and microscopic identification of eosinophils present in cerebrospinal fluid (CSF). 
It is caused by a variety of helminthic infections with the commonest being the ascariasis (Löffler's syndrome) angiostrongyliasis, gnathostomiasis toxocariasis, cysticercosis, schistosomiasis, baylisascariasis, and paragonimiasis (Bansal et al, 2014). El Bahnasawy et al. (2016) in Egypt reported that meningoencephalitis is an inflammation of the brain \& meninges (the lining of the brain and spinal cord). Based upon the history, physical examination and CSF findings, patients can often be classified as having probable bacterial or viral or parasitic meningitis or even non-infectious cause, although overlap can be frequent, particularly if bacterial meningitis has been partially treated with previous antibiotics. Organic brain syndromes are associated with both protozoan and helminthic infections; drugs side-effects commonly used to treat parasites may impair mood and cause anxiety, agitation or psychosis.

Imaging studies: Hepatic and cerebral lesions may be observed with ultrasonography, computed tomography (CT), and magnetic resonance imaging (Jain et al, 1994). In one review of radiographic changes associated with hepatic VLM, CT, and MRI showed multiple, ill-defined lesions, usually measuring 1.0 to $1.5 \mathrm{~cm}$ in diameter, scattered throughout the liver parenchyma ( $\mathrm{Za}-$ chariah et al, 1994). Lesions are usually oval but may be angular or trapezoid. The lesions differ from metastatic nodules in that they are usually uniform in size, non-spherical in shape, and are best seen on portal venous phase. Pulmonary VLM appear on CT as multifocal subpleural nodules with halo or ground-glass opacities and ill-defined margins (Schantz and Glickman, 1970).

Prognosis: For VLM is generally good; but, chronic one has potential correlations with both epilepsy \& cognitive delay. Complications rarely include blindness, severe neurologic disease, and death.

Treatment: The optimal treatment approach is uncertain and controlled data are limited. In general, individuals with mild sympt- oms due to toxocariasis do not require anthelminthic therapy; symptoms are usually selflimited and resolve within a few weeks (Stürchler et al, 1989). Eosinophilia may resolve much more slowly over many months, likely due to ongoing antigenic stimulation from dead larvae. In the setting of protracted symptoms, the reinfection possibility (such as from the continued ingestion of contaminated soil) must be considered.

For individuals with moderate to severe symptoms due to (VLM), it offered treatment with albendazole $(400 \mathrm{mg}$ orally twice daily for five days). In cases of severe respiratory, myocardial, or central nervous system (CNS) involvement, concomitant prednisone $(0.5$ to $1.0 \mathrm{mg} / \mathrm{kg} /$ day $)$ was warranted (Barisani et al, 2001).

Optimal approach to treatment of ocular larva migrans (OLM) is uncertain; there are some reports of response to albendazole treatment in adults with $800 \mathrm{mg}$ orally twice daily for two weeks; children: $400 \mathrm{mg}$ orally twice daily for two weeks (Small et al, 1989). This should be considered with concomitant prednisone $(1.5 \mathrm{mg} / \mathrm{kg}$ for adults) tapered over a few months. In complicated cases, surgical intervention may be warranted (Giuliari et al, 2011).

Mebendazole can alternate albendazole as 100 to $200 \mathrm{mg}$ orally twice/daily for five days (Magnaval, 1995), but albendazole is preferred, especially in OLM and neurologic infection, as it crosses blood-brain barrier. Ivermectin did not seem to be effective for toxocariasis (Magnaval, 1998). Diethylcarbamazine (DEC) (3 to $4 \mathrm{mg} / \mathrm{kg} /$ day for 21 days, starting at $25 \mathrm{mg}$ /day for adults) has been found to be effective in a small number of cases but with greater side-effects than albendazole.

Prevention: Good hygiene practices are important strategies to prevent zoonotic toxocariasis. Hand-washing must be encouraged after contact with pets or areas at high risk for the soil contamination, as playgrounds and sandboxes. Borecka and Kłapeć (2015) reported that to reduce the frequency of zo- 
onotic toxocariasis, some prophylaxis should be done, as public education of zoonotic diseases, systematic control of street animals, deworming of pets, cleaning pets' feces by the owners.

\section{Conclusion}

Toxocariasis is an infection transmitted from animals to humans (zoonosis) caused by the parasitic roundworms commonly found in the intestine of dogs (Toxocara canis) and cats ( $T$. cati). Larvae frequently localize in the liver; hepatic manifestations may include hepatomegaly or nodular lesions. Mild infection may be asymptomatic and only suspected by the finding of elevated blood eosinophilia. Heavy infection may result in fever, anorexia, malaise, irritability, hepatomegaly, and pruritic urticaria-like cutaneous lesions. Ocular larva migrans is due to larval localization in the eye and granulomatous response around the larva. Common symptoms are unilateral visual impairment and subsequent strabismus; complete visual loss can occur.

\section{Recommendations}

- VLM must be suspected in setting of compatible clinical manifestations together with leukocytosis, eosinophilia, and hypergammaglobulinemia (elevated serum levels of $\operatorname{IgE}, \operatorname{IgG}, \& \operatorname{IgE})$. In the appropriate clinical scenario, diagnosis can be confirmed by the ELISA to detect human IgG antibodies to Toxocara excretory/secretory antigens.

- Tuberculosis, toxocariasis and toxoplasmosis are among the common infectious lymphadenitis causes in children. So, when dealing with suspected OLM both Toxoplasma gondii and/or tuberculosis must be in mind - In general, individuals with mild symptoms due to toxocariasis do not require anthelminthic therapy; symptoms are usually self-limited. For individuals with moderate to severe symptoms albendazole (400mg orally twice daily for five days) was suggested. In cases of severe respiratory, myocardial, or CNS involvement, it was suggested concomitant treatment with prednisone (0.5$1.0 \mathrm{mg} / \mathrm{kg}$ /day) (Grade 2C).
- Good hygiene practices, timely disposal of pet feces, and routine deworming of pets are important strategies for prevention of toxocariasis in humans. Hand-washing must be encouraged after contact with pets or areas at high risk for soil contamination, such as playgrounds and sandboxes.

- Clean pets at least once a week. The feces should be either buried or bagged and disposed of in the trash. Hands must be washed after handling pet waste.

- Teach children that it is dangerous to eat the dirt or soil.

- Generally, Handwashing can help prevent illness. It involves five simple and effective steps (Wet, Lather, Scrub, Rinse, Dry) you can take to reduce the spread of diarrheal and respiratory illness so you can stay healthy. Regular handwashing, particularly before and after certain activities, is one of the best ways to remove germs, avoid getting sick, and prevent the spread of germs to others. It's quick, it's simple, and it can keep us all from getting sick. Handwashing is a win for everyone, except the germs.

\section{References}

Anderson, A, Fordham, L, Bula, ML, Blat, J, 2006: Visceral larval migrans masquerading as metastatic disease in a toddler with Wilms tumor. Pediatr. Radiol. 36, 3:265-67

Azam, D, Ukpai, OM, Said, A, et al, 2012: Temperature and the development and survival of infective Toxocara canis larvae. Parasitol. Res. 110:649-52.

Bansal, S, Gupta, M, Sharma, D, Bansal, S, 2014: A rare case of Ibuprofen-induced eosinophilic meningitis in a 13-year-old girl. Clin. Med. Insights Pediatr. 8:3-4.

Barisani-Asenbauer, T, Maca, SM, Hauff, W, et al, 2001: Treatment of ocular toxocariasis with albendazole. J. Ocul. Pharmacol. Ther. 17: 287-92.

Bede, O, Szénási, Z, Danka, J, et al, 2008: Toxocariasis associated with chronic cough in childhood: A longitudinal study in Hungary. J. Helminthol. 82:357-62.

Berrett, AN, Erickson, LD, Gale, SD, Stone, A, Brown, BL, et al, 2017: Toxocara seroprevalence and associated risk factors in the United States. Am. J. Trop. Med. Hyg. 97, 6:1846-50. 
Beshear, JR, Hendley, JO, 1973: Severe pulmonary involvement in visceral larva migrans. Am. J. Dis. Child. 125:599-604.

Borecka, A, Kłapeć, T, 2015: Epidemiology of human toxocariasis in Poland: A review of cases 1978-2009. Ann. Agric. Environ. Med. 22, 1: 28-31.

CDC, 2006: DPDx: Parasites and Health. Toxocariasis.

CDC, 2009: Division of Parasitic diseases. "Fact Sheet: Toxocariasis.

CDC, 2011: Ocular toxocariasis-United States, 2009-2010. MMWR Morb. Mortal. Wkly. Rep. 60:734-40.

CDC, 2019: Parasites-Toxocariasis (also known as Roundworm Infection.

Chan, PW, Anuar, AK, Fong, MY, Debruyne, JA, Ibrahim, J, 2001: Toxocara sero-prevalence and childhood asthma among Malaysian Children. Pediatr. Int. 43, 4:350-3.

Carvalho, EA, Rocha, RL, 2011: Toxocariasis: Visceral larva migrans in children. J. Pediatr. (Rio J). 87, 2:100-10.

Chuah, CT, Lim, MC, Seah, L, et al, 2006: Pseudoretinoblastoma in enucleated eyes of Asian patients. Singapore Med. J. 47:617-9.

Cooper, PJ, 2009: Interactions between helminth parasites and allergy. Curr Opin Allerg. Clin. Immunol. 9:29-32.

Cypess, RH, Karol, MH, Zidian, JL, et al, 1977: Larva-specific antibodies in patients with visceral larva migrans. J. Infect. Dis. 135:633-9.

de Visser, L, Rothova, A, de Boer, JH, et al, 2008: Diagnosis of ocular toxocariasis by establishing intraocular antibody production. Am. J. Ophthalmol.145:369-74.

Despommier, D, 2003: Toxocariasis: Clinical aspects, epidemiology, medical ecology, and molecular aspects. Clin. Microbiol. Rev. 16: 265-70.

Eberhardt, O, Bialek, R, Nägele, T, Dichgans, J, 2005: Eosinophilic meningomyelitis in toxocariasis: Case report and review of the literature. Clin. Neurol. Neurosurg. 107:432-9.

El-Bahnasawy, MMN, El Feky, MR, Morsy, ATA, Ismail, MAM, Morsy, TA, 2016: Egyptian eosinophilic and infectious meningoencephalitis and their impact on psychological aspects. J. Egypt. Soc. Parasitol. 46, 1: 67-80.

El-Tras, WF, Holt, HR, Tayel, AA, 2011: Risk of Toxocara canis eggs in stray \& domestic dog hair in Egypt. Vet. Parasitol. 178, 3/4:319-23
Fan, CK, Holland, CV, Loxton, K, Barghouth, U, 2015: Cerebral toxocariasis: Silent progression to neurodegenerative disorders? Clin. Micobiol. Rev. 28:663-86.

Fogt-Wyrwas, R, Jarosz, W, Mizgajska-Wiktor, H, 2007: Utilizing a polymerase chain reaction method for the detection of Toxocara canis and T. cati eggs in soil. J. Helminthol. 81:75-9.

Giuliari, GP, Ramirez, G, Cortez, R, 2011: Surgical treatment of ocular toxocariasis: Anatomic and functional results in 45 patients. Eur. J. Ophthalmol. 21:490-6.

Good, B, Holland, CV, Taylor, MR, et al, 2004: Ocular toxocariasis in schoolchildren. $\mathrm{Cl}$ in. Infect. Dis. 9:173-8.

Guneratne, R, Mendis, D, Bandara, T, Fernando, SD, 2011: Toxoplasma, Toxocara and tuberculosis co-infection in a four year old child BMC Pediatrics 11:44-8

Hotez, PJ, Wilkins, PP, 2009: Toxocariasis: America's most common neglected infection of poverty and a helminthiasis of global importance? PLoS Negl. Trop. Dis. 3:e400.

Ishibashi, H, Shimamura, R, Hirata, Y, et al, 1992: Hepatic granuloma in toxocaral infection: role of ultrasonography in hypereosinophilia. J. Clin. Ultrasound 20:204-10.

Jabbour, RA, Kanj, SS, Sawaya, RA, et al, 2011: Toxocara canis myelitis: Clinical features, magnetic resonance imaging (MRI) findings, and treatment outcome in 17 patients. Medicine (Baltimore) 90:337-42.

Jain, R, Sawhney, S, Bhargava, DK, et al, 1994: Hepatic granulomas due to visceral larva migrans in adults: Appearance on US and MRI. Abdom. Imaging 19:253-60.

Jones, JL, Moran DK, Won, K, Wilson, M, Schantz, PM, 2007: Toxoplasma gondii and Toxocara spp. Co-infection. Am. J. Trop. Mede. Hyg. 78, 1: 35-9.

Jones, JL, Kruszon-Moran, D, Won, K, et al, 2008: Toxoplasma gondii and Toxocara spp. coinfection. Am. J. Trop. Med. Hyg. 78: 35-40.

Kang, EJ, Choi, YJ, Kim, JS, Lee, BH, Kang, K, et al, 2014: Bladder and liver involvement of visceral larva migrans may mimic malignancy. Canc. Res. Treat. 46, 4:419-24

Kim, HG, Yang, JW, Hong, SC, Lee, YJ, Ju, YT, et al, 2018: Toxocara canis mimicking a metastatic omental mass from sigmoid colon cancer: A case report. Ann. Coloproctol. 34, 3: 160-8 
Lim, JH, 2008: Toxocariasis of the liver: Visceral larva migrans. Abdom. Imaging 33:151-6.

Macpherson, CN, 2013: The epidemiology and public health importance of toxocariasis: A zoonosis of global importance. Int. J. Parasitol. 43:999-1008.

Magnaval, JF, 1995: Comparative efficacy of diethylcarbamazine and mebendazole for the treatment of human toxocariasis. Parasitology 110, Pt 5:529-38.

Magnaval, JF, 1998: Apparent weak efficacy of ivermectin for treatment of human toxocariasis. Antimicrob. Agents Chemother. 42:2770-8. Magnaval, JF, Fabre, R, Maurières, $P$, et al, 1991: Application of the western blotting procedure for the immunodiagnosis of human toxocariasis. Parasitol. Res. 77:697-704.

Marx, C, Lin, J, Masruha, MR, et al, 2007: Toxocariasis of the CNS simulating acute disseminated encephalomyelitis. Neurology 69:80612.

Mohamed, AS, Moore, GE, Glickman, LT, 2009: Prevalence of intestinal nematode parasitism among pet dogs in the United States (20032006). J. Am. Vet. Med. Assoc. 234: 631-5.

Moreira, GM, TelmoPde, L, Mendonça, M, Moreira, AN, McBride, AJ, et al, 2014: Human toxocariasis: Current advances in diagnostics, treatment, \& intervention. Trends Parasitol. 30:456-64

Muñoz-Guzmán, MA, del Río-Navarro, BE, Valdivia, AG, Alba-Hurtado, F, 2010: The increase in seroprevalence to Toxocara canis in asthmatic children is related to cross-reaction with Ascaris suum antigens. Allergol. Immunopathol. (Madr) 38:115-9.

Nicoletti, A, 2013: Toxocariasis. Handb. Clin. Neurol. 114:217-28.

Ota, KV, Dimaras, H, Héon, E, et al, 2009: Toxocariasis mimicking liver, lung, and spinal cord metastases from retinoblastoma. Pediatr. Infect. Dis J. 28:252.

Roig, J, Romeu, J, Riera, C, et al, 1992: Acute eosinophilic pneumonia due to toxocariasis with bronchoalveolar lavage findings. Chest 102:2948.

Sakai, S, Shida, Y, Takahashi, N, et al, 2006: Pulmonary lesions associated with visceral larva migrans due to Ascaris suum or Toxocara canis: Imaging of six cases. AJR Am. J. Roentgenol. 186:1697-704.

Sander, JW, 2003: The epidemiology of epilepsy revisited. Curr. Opin. Neurol. 16:165-70

Schantz, PM, Glickman, LT, 1970: Toxocaral visceral larva migrans. N. Engl. J. Med. 298: 436-44.

Small, KW, McCuen, BW 2nd, de Juan, E Jr, Machemer, R, 1989: Surgical management of retinal traction caused by toxocariasis. Am. J. Ophthalmol. 108:10-8.

Snyder, C, 1961: Visceral larva migrans-ten years' experience. Pediatrics 28:85-92.

Stewart, JM, Cubillan, LD, Cunningham, ET Jr, 2005: Prevalence, clinical features, and causes of vision loss among patients with ocular toxocariasis. Retina 25:1005-11.

Stürchler, D, Schubarth, P, Gualzata, M, et al, 1989: Thiabendazole vs. albendazole in treatment of toxocariasis: a clinical trial. Ann. Trop. Med. Parasitol. 83:473-8.

Suh, LH, Sweeney, DA, Jun, AS, 2006: A 33year-old man with a white pupil. Clin. Infect. Dis. 43:1043.

Walsh, MG, 2011: Toxocara infection and diminished lung function in a nationally representative sample from the United States population. Int. J. Parasitol. 41:243-8.

Won, KY, Kruszon-Moran, D, Schantz, PM, Jones, JL, 2008: National seroprevalence, and risk factors for zoonotic Toxocara spp. infection. Am. J. Trop. Med. Hyg. 79:552-6.

Woodhall, DM, Fiore, AE, 2014: Toxocariasis: A Review for Pediatricians. J. Pediat. Infect. Dis. Soc. 3, 2:154-9

Youssef, AI, Uga, S, 2014: Review of parasitic zoonoses in Egypt. Trop. Med. Hlth. 42, 1:3-14.

Zachariah, SB, Zachariah, B, Varghese, R, 1994: Neuroimaging studies of cerebral "visceral larva migrans" syndrome. J. Neuroimaging 4: 39-44.

Zibaei, M, 2017: Helminth infections and cardiovascular diseases: Toxocara species is contributing to the disease. Curr. Cardiol. Rev. 13:5662. 